\title{
An Optical Approach to Microwave Frequency Measurement With Adjustable Measurement Range and Resolution
}

\author{
Xihua Zou and Jianping Yao, Senior Member, IEEE
}

\begin{abstract}
We propose an approach for the measurement of microwave frequency in the optical domain with adjustable measurement range and resolution. In the proposed approach, two optical wavelengths with a large wavelength spacing are modulated by an unknown microwave signal in a Mach-Zehnder modulator (MZM). The optical output from the MZM is sent to a dispersive fiber to introduce different chromatic dispersions, leading to different microwave power penalties. The two wavelengths are then separated, with the microwave powers measured by two photodetectors. A fixed relationship between the microwave power ratio and the microwave frequency is established. The microwave frequency is estimated by measuring the two microwave powers. The frequency measurement range and resolution can be adjusted by tuning the wavelength spacing. Different frequency measurement ranges and resolutions are demonstrated experimentally.
\end{abstract}

Index Terms-Fiber, microwave frequency measurement, microwave photonics, optical microwave signal processing.

\section{INTRODUCTION}

$\mathbf{M}$ ICROWAVE frequency measurement based on photonic techniques has attracted significant interest recently [1]-[7]. The primary advantages of microwave frequency measurement in the optical domain are the real-time and high-frequency operations which may not be achievable using conventional electronic techniques. In [1], an approach to microwave frequency measurement using an optical scanning receiver based on a tunable Fabry-Pérot interferometer was reported. Microwave frequency measurement can also be implemented based on a few other techniques, such as the use of an optical phased array [2], a high-resolution free-space diffraction grating [3], an array of phase-shifted gratings [4], or an integrated Bragg-grating combined with a Fresnel lens [5]. Recently, a technique for microwave frequency measurement

Manuscript received June 10, 2008; revised August 06, 2008. First published October 31, 2008; current version published November 21, 2008. This work was supported by The Natural Sciences and Engineering Research Council of Canada (NSERC). The work of X. Zou was supported by the China Scholarship Council.

X. Zou is with the Microwave Photonics Research Laboratory, School of Information Technology and Engineering, University of Ottawa, Ottawa, ON K1N 6N5, Canada, and also with the School of Information Science and Technology, Southwest Jiaotong University, Chengdu 610031, China (e-mail: xihuazou@site.uOttawa.ca).

J. Yao is with the Microwave Photonics Research Laboratory, School of Information Technology and Engineering, University of Ottawa, Ottawa, ON K1N 6N5, Canada (e-mail: jpyao@site.uOttawa.ca).

Color versions of one or more of the figures in this letter are available online at http://ieeexplore.ieee.org.

Digital Object Identifier 10.1109/LPT.2008.2005505

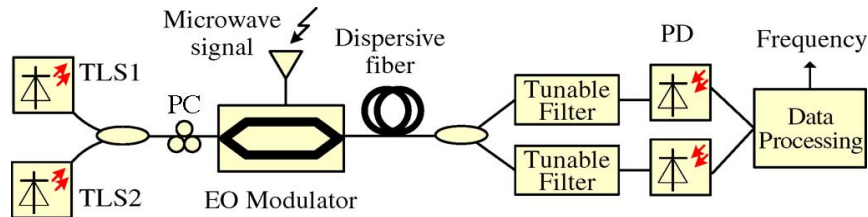

Fig. 1. Schematic diagram of the proposed frequency measurement system. PC: polarization controller. TLS: tunable laser source. PD: photodetectors.

was proposed by comparing the microwave powers of two intensity-modulated optical signals that experience different chromatic dispersions in a multichannel chirped fiber Bragg grating [6]. An approach based on optical power monitoring with a simplified system was also recently proposed [7].

A limitation of the approach in [7] is that the measurement range and resolution are fixed for a given system design. For many applications, however, it is highly desirable that the system is reconfigurable with adjustable measurement range and resolution. In [6], two different measurement ranges were obtained by using two different channels of a multichannel chirped fiber Bragg grating. The technique can be improved to have more measurement ranges if the multichannel chirped fiber Bragg grating is designed to have more channels.

In this letter, we propose a simple and flexible approach to microwave frequency measurement with an adjustable measurement range and resolution. The frequency is estimated by measuring the microwave powers of the two channels with different dispersion-induced power penalties. Thanks to a continuous distribution of the chromatic dispersion coefficient of the dispersive fiber, we can tune the wavelength spacing (several tens of nanometers) to get different dispersions, leading to an adjustable frequency measurement range and resolution. In addition, compared with a multichannel chirped fiber Bragg grating, a dispersive fiber has less dispersion ripples, which can provide a better measurement accuracy.

\section{PRINCIPLE}

As shown in Fig. 1, the proposed system consists of two tunable laser sources (TLSs), a Mach-Zehnder modulator (MZM), a length of dispersive fiber, two tunable optical filters, and two photodetectors (PDs). A microwave signal with its frequency to be measured is applied to the MZM to intensity-modulate the two optical wavelengths. Since the microwave signal is usually at a low power level, small signal modulation is considered. The two intensity-modulated lightwaves are then sent to the dispersive fiber. Since the chromatic dispersions are different for the two wavelengths, different dispersion-induced power penalties 
would result. The two wavelengths are then separated by the optical filters. The output microwave powers are measured at the outputs of the two PDs.

Considering the dispersion-induced power penalties [8], the microwave powers at the outputs of the two PDs are given by

$$
\begin{aligned}
& P_{1}=\Re_{1}\left[\cos \left(\pi \chi_{1} \lambda_{1}^{2} f^{2} / c\right)\right]^{2} \\
& P_{2}=\Re_{2}\left[\cos \left(\pi \chi_{2} \lambda_{2}^{2} f^{2} / c\right)\right]^{2}
\end{aligned}
$$

where $f$ is the microwave frequency, $c$ is the light velocity in vacuum, $\lambda_{i}, i=1,2$, are the two wavelengths, $\chi_{i}$ are the total chromatic dispersions of the dispersive fiber for $\lambda_{i}, \Re_{i}$ denote the losses of the two channels including the losses in the dispersive fiber, the MZM, and the PD. The modulation in the MZM should be chirp free or the expression in (1) has to be revised to incorporate the chirp component generated during the modulation [6], to reduce frequency measurement errors.

Although the losses in the dispersive fiber for $\lambda_{1}$ and $\lambda_{2}$ are different, the loss difference can be compensated by adjusting the powers of the TLSs. Therefore, we have $\Re_{1}=\Re_{2}$. Based on (1), we have the following power ratio:

$$
\gamma=\frac{P_{1}}{P_{2}}=\frac{\left[\cos \left(\pi \chi_{1} \lambda_{1}^{2} f^{2} / c\right)\right]^{2}}{\left[\cos \left(\pi \chi_{2} \lambda_{2}^{2} f^{2} / c\right)\right]^{2}} .
$$

As can be seen from (2), the microwave frequency can be calculated if the power ratio is known. We should note that a small wavelength spacing (i.e., several nanometers) would lead to a power ratio approach to unity, which would significantly reduce the estimation resolution. To get a higher measurement resolution, a large wavelength spacing (usually several tens of nanometers) is required.

The key novelty of this technique is the employment of the dispersive fiber, which results in different microwave power penalties for the two wavelengths, leading to a nonunity power ratio. The frequency measurement range and resolution can be adjusted if the wavelength spacing is tuned. Therefore, to have a specific measurement range or resolution, one can simply tune the wavelength spacing. In addition, the use of a dispersive fiber would have much smaller dispersion ripples compared to the use of a multichannel chirped fiber Bragg grating [6], which would ensure a better measurement performance.

\section{EXPERIMENT AND DISCUSSION}

A proof-of-concept experiment is performed. A $20-\mathrm{km}$ single-mode fiber (SMF) is employed as the dispersive fiber. The dispersions of the SMF for six different wavelengths at $1470,1500,1520,1550,1580$, and $1600 \mathrm{~nm}$ are 234.5, 273, $298,335,370$, and $393 \mathrm{ps} / \mathrm{nm}$, measured by the phase-shift method. When the TLS is operating at these wavelengths, the corresponding power distributions are measured by a vector network analyzer (Agilent, E8364A), as shown in Fig. 2. The location of the first notch is determined by the total dispersion and the wavelength, and thus determines the measurement range and resolution.

In the experiment, the two wavelengths are first set at 1470 and $1600 \mathrm{~nm}$, the powers at different microwave frequencies are measured with the power ratio being calculated. As can be seen from Fig. 3(a), the measured power ratios agree well with the theoretical values. Based on the power ratios, the microwave frequencies are calculated, as shown in Fig. 3(b). To have a better

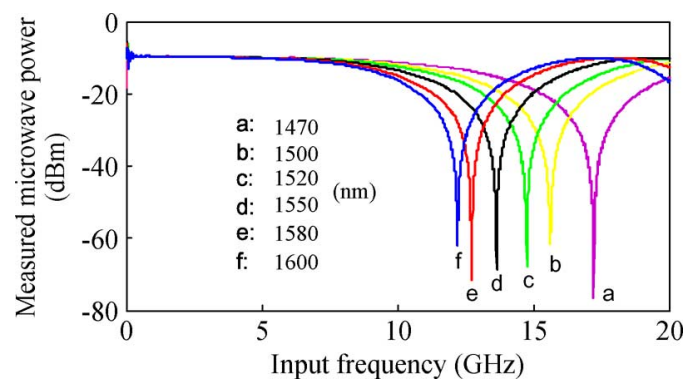

Fig. 2. Measured power distributions for different wavelengths.
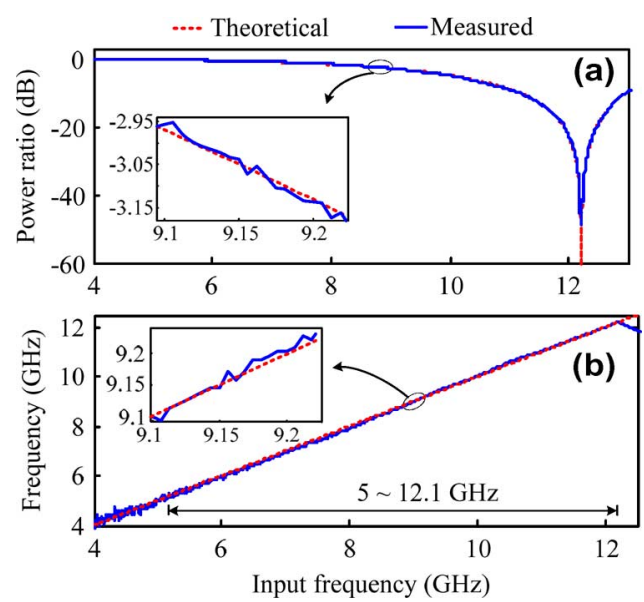

Fig. 3. (a) Power ratio and (b) frequency measurement range for the system operating at 1470 and $1600 \mathrm{~nm}$.

observation of the measured frequencies, two zoom-in views are shown as two insets in Fig. 3. Small measurement errors are observed. The measurement errors are mainly due to the system noise and power fluctuations of the laser sources. For a given measurement error, for example $\pm 0.1 \mathrm{GHz}$, the measurement range is from 5 to $12.1 \mathrm{GHz}$. Beyond this measurement range, especially at the low-frequency band, the measurement errors are significantly increased.

Two important features are associated with the proposed technique. First, the technique provides the ability to flexibly adjust the measurement range and resolution. Since the measurement range is limited by the dispersion difference between the two wavelengths, by tuning the wavelength spacing, the measurement range is thus changed. For instant, if the longer wavelength of $1600 \mathrm{~nm}$ is tuned to $1520 \mathrm{~nm}$ while keeping the shorter wavelength of $1470 \mathrm{~nm}$ unchanged, a new measurement range of $9-14.7 \mathrm{GHz}$ is achieved for the same measurement error of $\pm 0.1 \mathrm{GHz}$, as shown in Fig. 4. Second, the resolution can also be adjusted. Usually there is a trade-off between the measurement range and resolution: a higher resolution is achieved at the cost of a relatively smaller measurement range. As shown in Fig. 5, a significantly reduced measurement error of $\pm 0.02 \mathrm{GHz}$ is achieved for a smaller measurement range of $11-13.6 \mathrm{GHz}$, when the two wavelengths are set at 1520 and $1550 \mathrm{~nm}$ with a smaller wavelength spacing. To improve the measurement resolution while maintaining the same measurement range, a solution is to add a third wavelength. The fundamental principle is as follows: the use of three wavelengths would lead to two separate measurement 


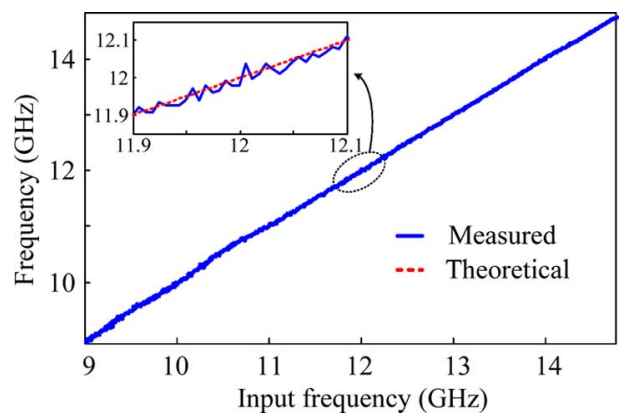

Fig. 4. Measurement range when the system is operating at 1470 and $1520 \mathrm{~nm}$.

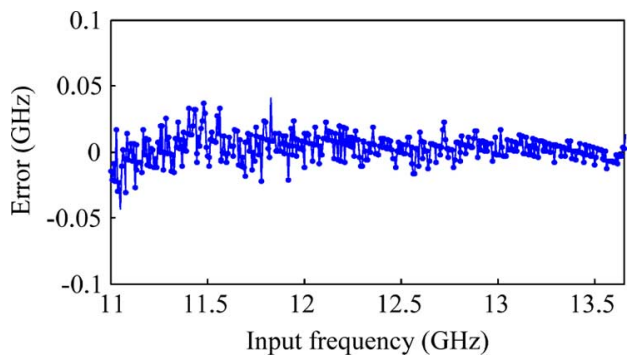

Fig. 5. Reduced measurement error of $\pm 0.02 \mathrm{GHz}$ when the system is operating at 1520 and $1550 \mathrm{~nm}$ for a measurement range of $11-13.6 \mathrm{GHz}$.

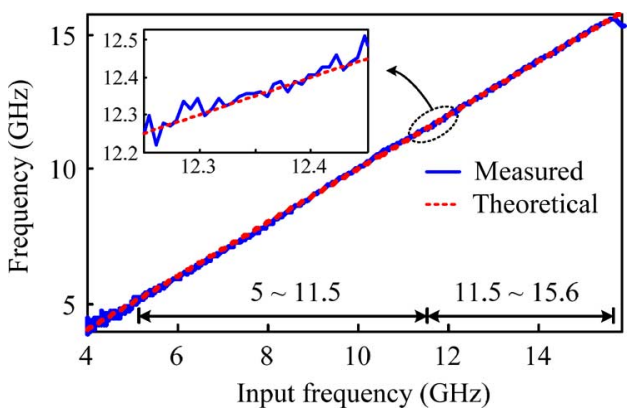

Fig. 6. Measurement range for three wavelengths.

ranges with each having a higher resolution. The combination of the two measurement ranges would make a larger measurement range while keeping a better measurement resolution. In Fig. 6, for example, three wavelengths at 1470, 1500, and $1600 \mathrm{~nm}$ are used. For a measurement error of $\pm 0.1 \mathrm{GHz}$, the two wavelengths at 1470 and $1600 \mathrm{~nm}$ would provide a range of 5-11.5 GHz, while the wavelengths at 1470 and $1500 \mathrm{~nm}$ would offer another range of $11.5-15.6 \mathrm{GHz}$. Hence, the total measurement range is $5-15.6 \mathrm{GHz}$ with an error of $\pm 0.1 \mathrm{GHz}$.

As discussed earlier, the system noise has an impact on the measurement resolution. Based on the central limit theorem, we assume that the system noise is a Gaussian-distribution noise. To ensure a measurement error of $\pm 0.1 \mathrm{GHz}$, the signal-to-noise ratio should be greater than $14 \mathrm{~dB}$ when the two wavelengths are set as 1470 and $1520 \mathrm{~nm}$. The minimum microwave power required can be calculated if the noise power is known. For instance, the required input microwave power should be $-23 \mathrm{dBm}$ for a $10-\mathrm{GHz}$ microwave in our setup where the noise power is $-95 \mathrm{dBm}$.

This technique can find applications in a warfare system where a wideband and real-time frequency measurement is required. The difference between this technique and the conventional frequency measurement techniques using an electronic spectrum analyzer is that an electronic spectrum analyzer usually performs a narrowband frequency measurement at a time. Therefore, the proposed technique is suitable for instantaneous frequency measurement.

\section{CONCLUSION}

We have proposed a novel and simple approach for microwave frequency measurement in the optical domain. The key device in the system is the dispersive fiber, which was used to introduce different chromatic dispersions, leading to different power penalties. The microwave frequency was calculated by measuring the microwave powers, thanks to a fixed relationship between the power ratio and the microwave frequency. In addition to the simplicity, the proposed technique has a significant advantage: it has an adjustable measurement range and resolution. There is a trade-off between the measurement range and resolution. However, the trade-off could be eliminated by using three or more wavelengths, which can provide a significantly improved measurement resolution while maintaining a large measurement range. An experiment was performed. Different frequency measurement ranges and resolutions were experimentally demonstrated.

\section{REFERENCES}

[1] S. T. Winnall and A. C. Lindsay, "A Fabry-Pérot scanning receiver for microwave signal processing," IEEE Trans. Microw. Theory Tech., vol. 47, no. 7, pp. 1385-1390, Jul. 1999.

[2] J. M. Heaton, C. D. Waston, S. B. Jones, M. M. Bourke, C. M. Boyne, G. W. Smith, and D. R. Wight, "Sixteen channel (1 to $16 \mathrm{GHz}$ ) microwave spectrum analyzer device based on a phased array of GaAs/AlGaAs electro-optic waveguide delay lines," Proc. SPIE, vol. 3278, pp. 245-251, 1998.

[3] W. Wang, R. L. Davis, T. J. Jung, R. Lodenkamper, L. J. Lembo, J. C. Brock, and M. C. Wu, "Characterization of a coherent optical RF channelizer based on a diffraction grating," IEEE Trans. Microw. Theory Tech., vol. 49, no. 10, pp. 1996-2001, Oct. 2001.

[4] D. B. Hunter, L. G. Edvell, and M. A. Englund, "Wideband microwave photonic channelised receiver," in Proc. Int. Topical Meeting Microwave Photonics, Oct. 2005, pp. 249-251.

[5] S. T. Winnall, A. C. Lindsay, M. W. Austin, J. Canning, and A. Mitchell, "A microwave channelizer and spectroscope based on an integrated optical Bragg-grating Fabry-Pérot and integrated hybrid Fresnel lens system," IEEE Trans. Microw. Theory Tech., vol. 54, no. 2, pp. 868-892, Feb. 2006

[6] L. V. T. Nguyen and D. B. Hunter, "A photonic technique for microwave frequency measurement," IEEE Photon. Technol. Lett., vol. 18, no. 10, pp. 1188-1190, May 15, 2006.

[7] H. Chi, X. Zou, and J. P. Yao, "An approach to the measurement of microwave frequency based on optical power monitoring," IEEE Photon. Technol. Lett., vol. 20, no. 14, pp. 1249-1251, Jul. 15, 2008.

[8] G. H. Smith, D. Novak, and Z. Ahmed, "Overcoming chromatic-dispersion effects in fiber-wireless systems incorporating external modulators," IEEE Trans. Microw. Theory Tech., vol. 45, no. 8, pp. 1410-1415, Aug. 1997. 